\title{
Soft-resummation corrections to parton distributions
}

\author{
Gennaro Corcella* \\ Dipartimento di Fisica \\ Università di Roma 'La Sapienza' \\ P.le A.Moro 2, I-00185 Roma, Italy \\ E-mail: Gennaro.Corcella@romal.infn.it

\section{Lorenzo Magnea} \\ Dipartimento di Fisica Teorica \\ Università di Torino and INFN, Sezione di Torino \\ Via P. Giuria, I-10125 Torino, Italy \\ E-mail: magnea@to.infn.it
}

We investigate the impact of large- $x$ resummation on parton distribution functions. We consider structure function data from the NuTeV, NMC and BCDMS experiments and extract NLO and NLL-resummed parton densities. Our analysis shows that soft resummation needs to be included for the sake of obtaining a precision level better than $10 \%$ at $x>0.5$ and moderate values of $Q^{2}$.

Diffraction 06, International Workshop on Diffraction in High-Energy Physics September 5-10, 2006

Adamantas, Milos island, Greece

${ }^{*}$ Speaker. 
An accurate knowledge of parton distribution functions (PDF's) will be essential in order to achieve the current precision goals at present and future hadron colliders, and in particular at the LHC (see, e.g., Refs. [1, 2]). In this paper we shall present a simple analysis using Deep Inelastic Scattering (DIS) structure function data to extract next-to-leading order (NLO) and large- $x$ resummed parton distribution functions.

Structure functions $F_{i}\left(x, Q^{2}\right)$ are given by the convolution of partonic coefficient functions and PDF's. Up to now, PDF analyses have been performed at fixed order in perturbative QCD: using, e.g., NLO coefficient functions one extracts NLO PDF's. The coefficient functions, however, present terms that are enhanced for large values of the Bjorken variable $x$ : the limit $x \rightarrow 1$ corresponds to soft- or collinear-gluon radiation. These contributions need to be resummed to all orders to improve the validity of the perturbative prediction. Large- $x$ resummation for the DIS coefficient function was performed in $[3,4]$ in the massless approximation, and in $[5,6]$ with the inclusion of quark-mass effects, which are relevant when the scale of the process is comparable with the heavy-quark mass.

Soft resummation is typically performed in Mellin moment space, where large- $x$ terms correspond, at $\mathscr{O}\left(\alpha_{s}\right)$, to single $\left(\alpha_{s} \ln N\right)$ and double $\left(\alpha_{s} \ln ^{2} N\right)$ logarithms of the variable $N$. Such logarithms exponentiate in a Sudakov-like form factor: in the next-to-leading logarithmic (NLL) approximation, which we shall adopt hereafter, terms $\mathscr{O}\left(\alpha_{s}^{n} \ln ^{n+1} N\right)(\mathrm{LL})$ and $\mathscr{O}\left(\alpha_{s}^{n} \ln ^{n} N\right)$ (NLL) are included in the Sudakov exponent. Using large- $x$ resummed coefficient functions, we can extract resummed PDF's from DIS structure function data. Large- $x$ data are typically provided by fixed-target experiments: we shall analyse charged-current (CC) data from neutrino-iron scattering, collected by the NuTeV experiment [7], and neutral-current (NC) data from the NMC [8] and BCDMS $[9,10]$ collaborations.

In particular, we shall use NuTeV data on $F_{2}(x)$ and $x F_{3}(x)$ at $Q^{2}=31.62 \mathrm{GeV}^{2}$ and 12.59 $\mathrm{GeV}^{2}$ : in fact, as discussed in [6], $Q^{2}$ should not be too large for the resummation to have an impact. Fitting the data using NLO and NLL-resummed CC coefficient functions, we shall be able to extract NLO and NLL-resummed quark distributions. Since $F_{2}$ contains a gluon-initiated contribution $F_{2}^{g}$, which is not soft-enhanced and is very small at large $x$, we take $F_{2}^{g}$ from a global fit, e.g. the NLO set CTEQ6M [11], and fit only the quark-initiated $F_{2}^{q}$. We choose a simple parametrization of the form $F_{2}^{q}(x)=F_{2}(x)-F_{2}^{g}(x)=A x^{-\alpha}(1-x)^{\beta}(1+b x) ; x F_{3}(x)=C x^{-\rho}(1-x)^{\sigma}(1+k x)$. The best-fit parameters and the $\chi^{2}$ per degree of freedom from the fit were presented in [12]. In Figs. 1 and 2, we just show the NuTeV data on $F_{2}(x)$ and $x F_{3}(x)$ at $Q^{2}=12.59 \mathrm{GeV}^{2}$ and $31.62 \mathrm{GeV}^{2}$, along with our best-fit curves.

For the sake of extracting individual quark distributions, it is mandatory to use more data. We consider the NC structure functions measured by the NMC and BCDMS collaboration, and make use of the parametrization of the nonsinglet structure function $F_{2}^{\mathrm{ns}}=F_{2}^{p}-F_{2}^{D}$ determined by the NNPDF collaboration [13]. Such a parametrization is obtained after training neural networks with Monte Carlo copies of the data, and includes all information concerning errors and correlations. The resulting structure functions are therefore an unbiased representation of the data. Fig. 3 shows $F_{2}^{\text {ns }}\left(x, Q^{2}\right)$, computed with the neural network parametrization at our chosen values of $Q^{2}$, for $x=n / 40, n=1, \ldots, 39$. The central values are averages of the results yielded by one thousand neural networks, while the error bars are the corresponding standard deviations. We learn from Fig. 3 that the the errors are quite large, which is due to the fact that $F_{2}^{\text {ns }}\left(x, Q^{2}\right)$ is the difference 

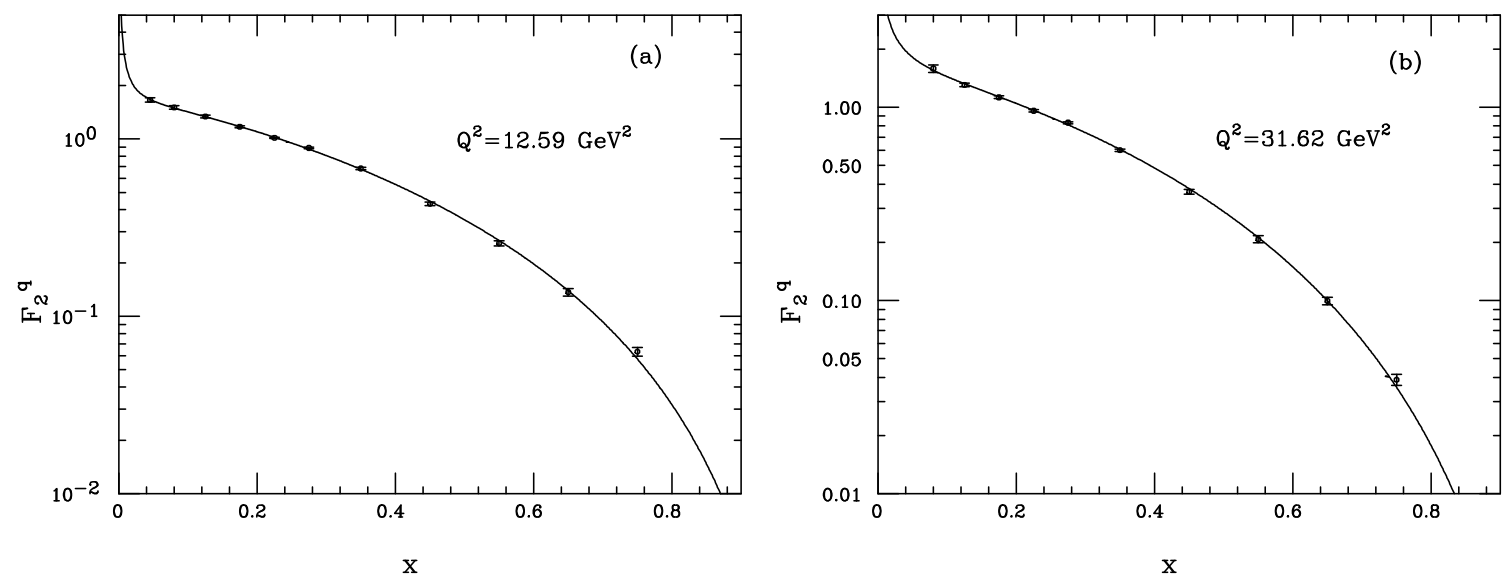

Figure 1: NuTeV data and results yielded by our best fi t for the structure function $F_{2}^{q}$ at $Q^{2}=12.59 \mathrm{GeV}^{2}$ (a) and $31.62 \mathrm{GeV}^{2}$ (b).
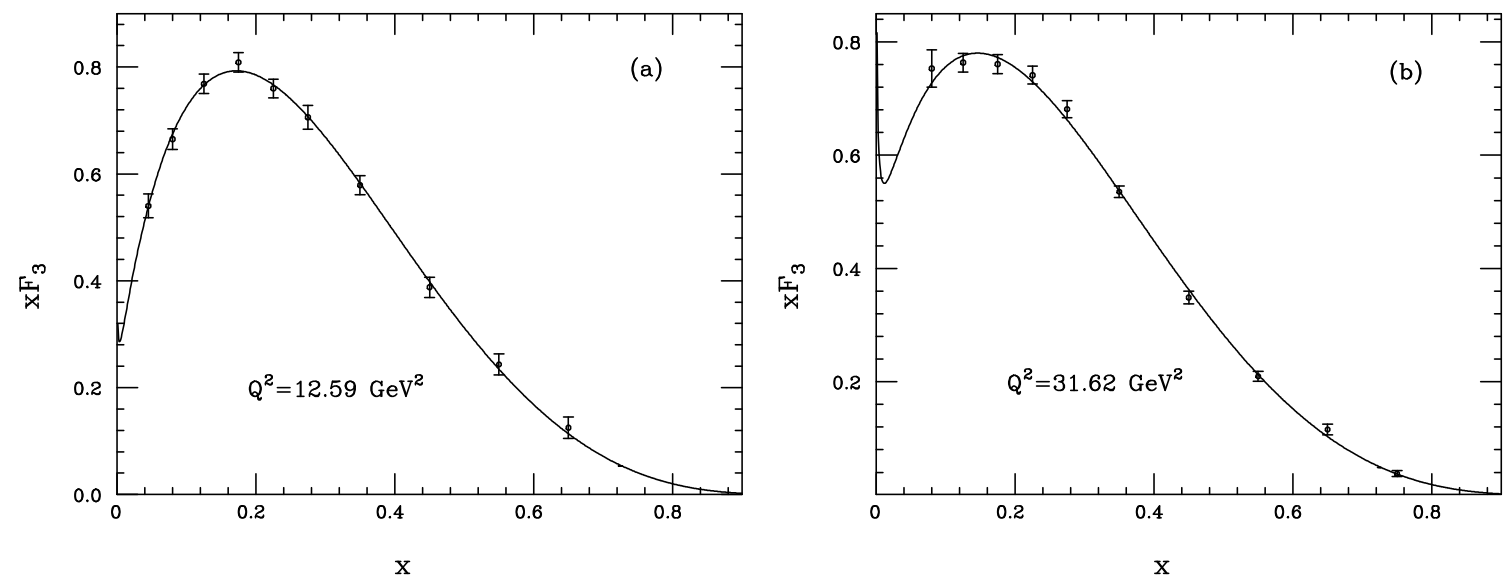

Figure 2: As in Fig. 1, but for the structure function $x F_{3}$.
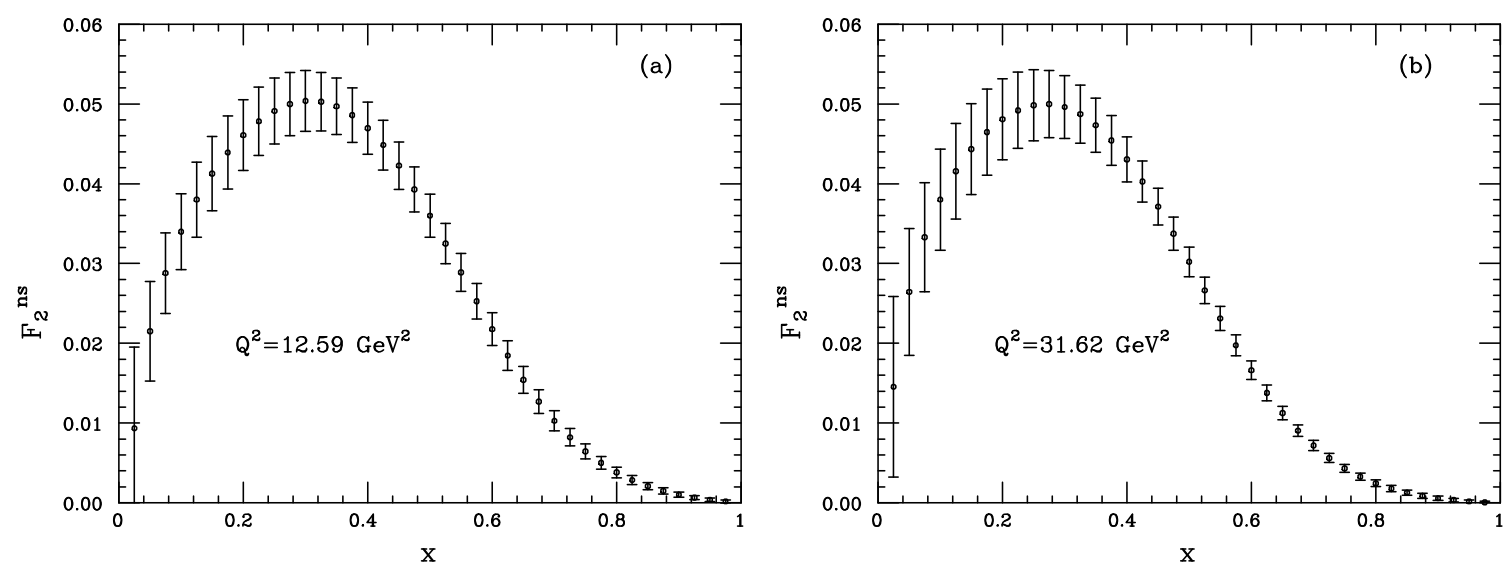

Figure 3: A sampling of the neural parametrization of NMC and BCDMS data for $F_{2}^{\text {ns }}\left(x, Q^{2}\right)$ at $Q^{2}=$ $12.59 \mathrm{GeV}^{2}$ (a) and at $Q^{2}=31.62 \mathrm{GeV}^{2}$ (b), from the NNPDF Collaboration [13]. 
between proton and deuteron structure functions, resulting in a loss of precision.

Writing $F_{2}, x F_{3}$ and $F_{2}^{\text {ns }}$ in terms of their parton content, we can extract NLO and NLLresummed quark densities, according to whether we use in the fit NLO or NLL coefficient functions. To simplify our analysis, we assume that the sea is isospin-symmetric, i.e. $s=\bar{s}$ and $\bar{u}=\bar{d}$, we neglect the charm-quark density, and impose a proportionality relation $\bar{s}=\kappa \bar{u}$. We can thus obtain a system of three equations which can be solved in terms of $u, d$ and $s$, as done in [12]. We begin by working in $\mathrm{N}$-space, where the resummation has a simpler form and parton distributions are just the ratio of the appropriate structure function and coefficient function. The results can then be inverted back to $x$-space using, e.g., a simple power law such as $q(x)=D x^{-\gamma}(1-x)^{\delta}$.

Figs. 4-5 exhibit the impact of large- $x$ resummation on the up-quark distribution at $Q^{2}=12.59$ and $31.62 \mathrm{GeV}^{2}$, in $N$ - and $x$-spaces, after setting $\kappa=1 / 2$ in the relation between $\bar{s}$ and $\bar{u}$. We quote in Table 1 our best-fit results for $D, \gamma$ and $\delta$, and the corresponding $\chi^{2} /$ dof.

The impact of the resummation is indeed remarkable at large $N$ and $x$ : the coefficient function and its moments are enhanced, and therefore the quark densities are suppressed. Such an effect is larger at $Q^{2}=12.59 \mathrm{GeV}^{2}$ : this is just a consequence of the growth of $\alpha_{S}$ as the scale becomes smaller. In Fig. 5 we also present the up-quark density according to the MRST2001 NLO set [14]. Given the different approximations which we made in our analysis, we do not expect that our results should agree with the MRST2001 global fit. However, it is remarkable that at low $x$ the MRST2001 up-quark distribution is within the error range of the densities extracted form our fit. At large $x$ it looks closer to our NLL-resummed PDF rather than to the NLO one. In fact, as observed in [15], the MRST2001 set was fitted to CCFR structure function data [16], which are lower than NuTeV at large $x$. It is therefore reasonable that the NLO MRST2001 PDF's be lower than the NLO ones which we extracted from a fit to $\mathrm{NuTeV}$, and therefore closer to our resummed PDF's. The discrepancy between NuTeV and CCFR at large $x$ is now described as understood [7]. In principle, even the $d$ and $s$ densities should be affected by the resummation; we found, however, that, within our simple model, the errors on such PDF's are too large for us to be able to discriminate soft-gluon effects.

\begin{tabular}{clccc}
\hline$Q^{2}$ & PDF & $D$ & $\gamma$ & $\delta$ \\
\hline 12.59 & NLO & $3.025 \pm 0.534$ & $0.418 \pm 0.101$ & $3.162 \pm 0.116$ \\
& RES & $4.647 \pm 0.881$ & $0.247 \pm 0.109$ & $3.614 \pm 0.128$ \\
31.62 & NLO & $2.865 \pm 0.420$ & $0.463 \pm 0.086$ & $3.301 \pm 0.098$ \\
& RES & $3.794 \pm 0.583$ & $0.351 \pm 0.090$ & $3.598 \pm 0.104$ \\
\hline
\end{tabular}

Table 1: Best fi t values and errors for the up-quark $x$-space parametrization, at the chosen values of $Q^{2}$.

Fig. 6 presents the ratio $\Delta u(x)=\left(u_{\mathrm{NLO}}(x)-u_{\mathrm{res}}(x)\right) / u_{\mathrm{NLO}}(x)$, at both values of $Q^{2}$, for the central values of the best-fit parameters, as quoted in Table 1 . The suppression of the resummed up quark distribution with respect to the NLO one is about $5 \%$ at $x \simeq 0.58,10 \%$ at $x \simeq 0.65$ and $20 \%$ at $x \simeq 0.75$ for $Q^{2}=12.59 \mathrm{GeV}^{2}$, while for $Q^{2}=31.62 \mathrm{GeV}^{2}$ the same factors correspond to $x \simeq 0.61, x \simeq 0.69$ and $x \simeq 0.8$. 

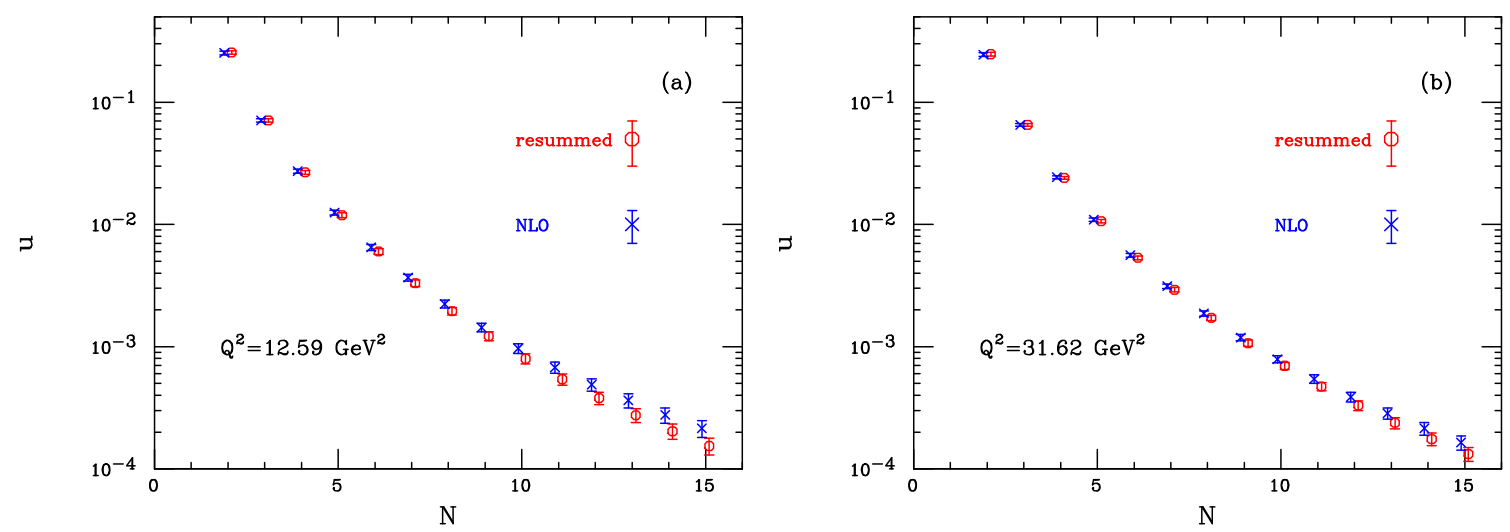

Figure 4: NLO and resummed moments of the up quark distribution at $Q^{2}=12.59 \mathrm{GeV}^{2}$ (a) and 31.62 $\mathrm{GeV}^{2}$.
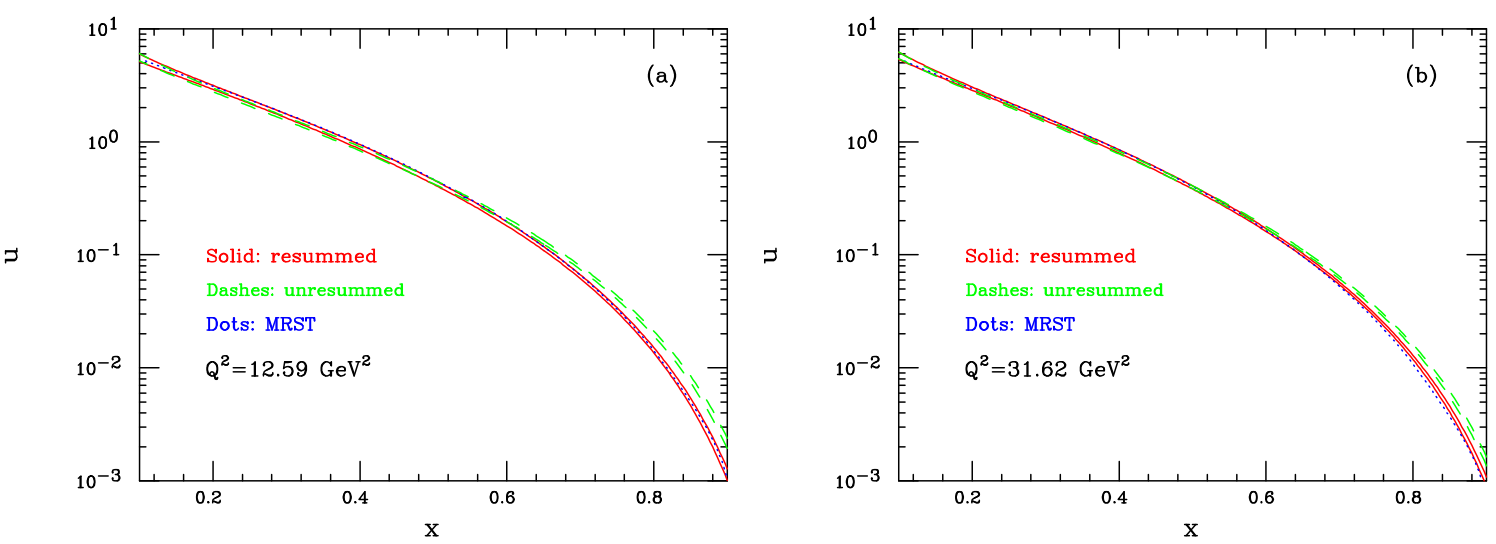

Figure 5: NLO and NLL up-quark density in $x$-space at $Q^{2}=12.59 \mathrm{GeV}^{2}$ (a) and $31.62 \mathrm{GeV}^{2}$ (b). Plotted are the statistical bands at one-standard-deviation confi dence level. For the sake of comparison, we present also the MRST2001 result.

We finally check that our results at the two values of $Q^{2}$ are consistent with NLO DGLAP evolution. In Fig. 7 we present, in Mellin space, the NLO and NLL-resummed results obtained from a fit of the data at $12.59 \mathrm{GeV}^{2}$ and from the evolution of the moments fitted at $31.62 \mathrm{GeV}^{2}$. The results are consistent just within one standard deviation; it is nonetheless remarkable that the evolution of the resummed moments is less consistent than the NLO one. This feature may deserve further thoughts: one may have to investigate more in depth the DGLAP evolution equations at large $x$ to draw a firm conclusion.

In summary, we have analysed large- $x$ DIS structure function data from NuTeV, BCDMS and NMC experiments, and extracted NLO and NLL-resummed parton densities. We have estimated that the impact of soft gluon resummation on valence quark densities can be as much as $10-20 \%$ at $x>0.5$ and moderate $Q^{2}$. We believe that it would be useful to include soft resummation in future PDF global analyses, in order to obtain better precisions in the measurements which are planned for the LHC, especially the ones requiring great accuracy, and for which a good theoretical prediction is available, such as weak-boson production through the Drell-Yan process. 

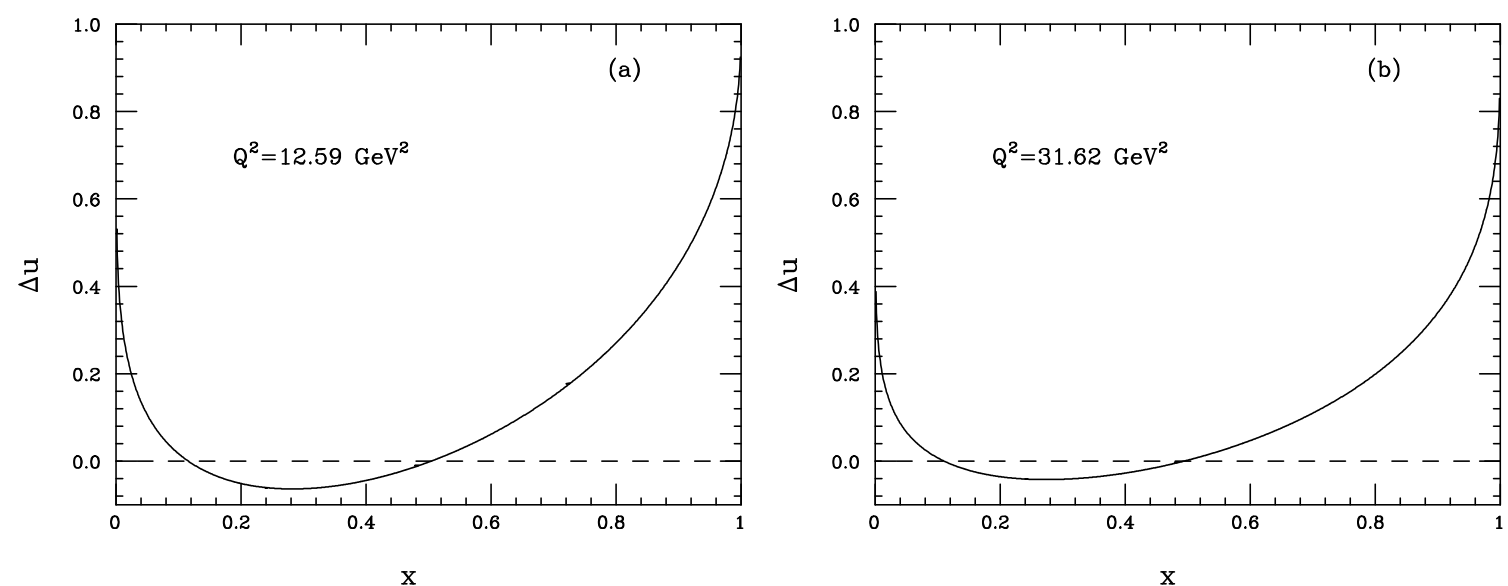

Figure 6: Relative effect of large- $x$ resummation on the up-quark density at $Q^{2}=12.59 \mathrm{GeV}^{2}$ (a) and 31.62 $\mathrm{GeV}^{2}$.
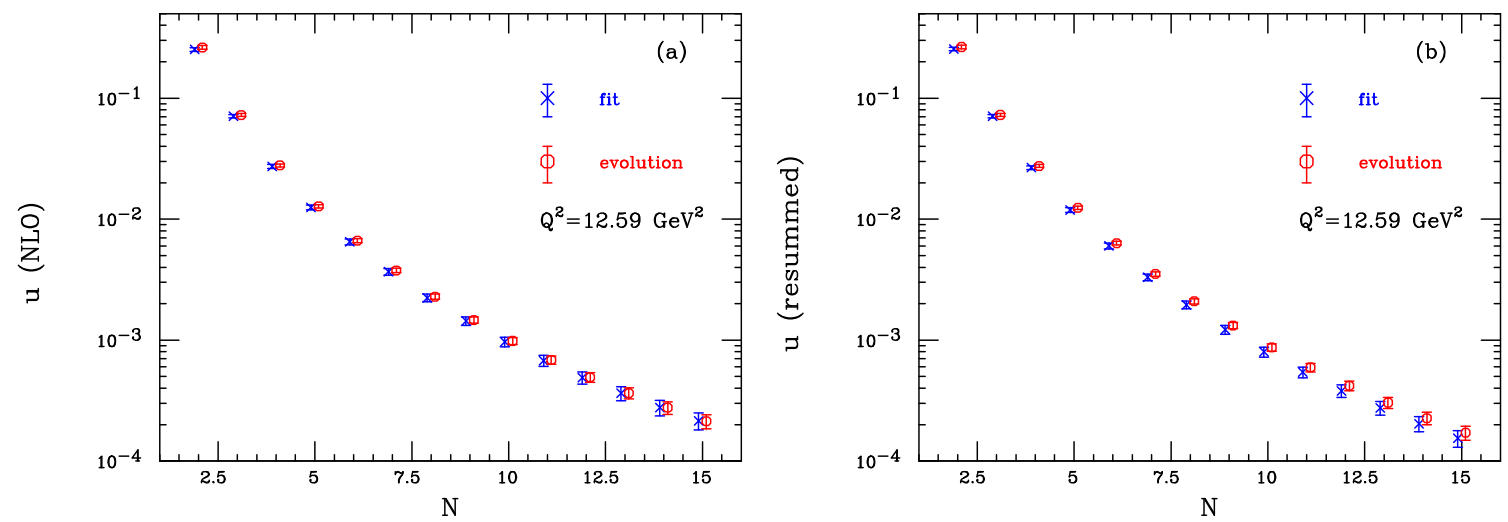

Figure 7: Moments of the up-quark distributions fi tted at $Q^{2}=12.59 \mathrm{GeV}^{2}$ moments obtained via NLO evolution from a fit at $Q^{2}=31.62 \mathrm{GeV}^{2}$.

\section{References}

[1] S. Alekhin et al., arXiv:hep-ph/0601013.

[2] C. Buttar et al., arXiv:hep-ph/0604120.

[3] G. Sterman, Nucl. Phys. B 281 (1987) 310.

[4] S. Catani and L. Trentadue, Nucl. Phys. B 327 (1989) 323.

[5] E. Laenen and S. O. Moch, Phys. Rev. D 59 (1999) 034027 [arXiv:hep-ph/9809550].

[6] G. Corcella and A. D. Mitov, Nucl. Phys. B 676 (2004) 346 [arXiv:hep-ph/0308105].

[7] M. Tzanov et al. [NuTeV Collaboration], Phys. Rev. D 74 (2006) 012008 [arXiv:hep-ex/0509010].

[8] M. Arneodo et al. [New Muon Collaboration], Nucl. Phys. B 483 (1997) 3 [arXiv:hep-ph/9610231].

[9] A. C. Benvenuti et al. [BCDMS Collaboration], Phys. Lett. B 223 (1989) 485.

[10] A. C. Benvenuti et al. [BCDMS Collaboration], Phys. Lett. B 237 (1990) 592. 
[11] J. Pumplin, D. R. Stump, J. Huston, H. L. Lai, P. Nadolsky and W. K. Tung, JHEP 0207 (2002) 012 [arXiv:hep-ph/0201195].

[12] G. Corcella and L. Magnea, Phys. Rev. D 72 (2005) 074017 [arXiv:hep-ph/0506278].

[13] L. Del Debbio, S. Forte, J. I. Latorre, A. Piccione and J. Rojo [NNPDF Collaboration], JHEP 0503 (2005) 080 [arXiv:hep-ph/0501067].

[14] A. D. Martin, R. G. Roberts, W. J. Stirling and R. S. Thorne, Phys. Lett. B 531 (2002) 216 [arXiv:hep-ph/0201127].

[15] G. Corcella and L. Magnea, AIP Conf. Proc. 792 (2005) 303 [arXiv:hep-ph/0507042].

[16] U. K. Yang et al. [CCFR/NuTeV Collaboration], Phys. Rev. Lett. 86 (2001) 2742 [arXiv:hep-ex/0009041]. 